\title{
Virtual Antenna Array Analysis for MIMO Synthetic Aperture Radars
}

\author{
Wen-Qin Wang \\ School of Communication and Information Engineering, University of Electronic Science and Technology of China (UESTC), \\ Chengdu 611731, China
}

Correspondence should be addressed to Wen-Qin Wang, wqwang@uestc.edu.cn

Received 17 July 2011; Revised 9 October 2011; Accepted 18 October 2011

Academic Editor: Wenhua Chen

Copyright ( 92012 Wen-Qin Wang. This is an open access article distributed under the Creative Commons Attribution License, which permits unrestricted use, distribution, and reproduction in any medium, provided the original work is properly cited.

Multiple-input multiple-output (MIMO) synthetic aperture radar (SAR) that employs multiple antennas to transmit orthogonal waveforms and multiple antennas to receive radar echoes is a recently proposed remote sensing concept. It has been shown that MIMO SAR can be used to improve remote sensing system performance. Most of the MIMO SAR research so far focused on signal/data models and corresponding signal processing algorithm. Little work related to MIMO SAR antenna analysis can be found. One of the main advantages of MIMO SAR is that the degrees of freedom can be greatly increased by the concept of virtual antenna array. In this paper, we analyze the virtual antenna array for MIMO SAR high-resolution wide-swath remote sensing applications. The one-dimensional uniform and nonuniform linear antenna arrays are investigated and their application potentials in high-resolution wide-swath remote sensing are introduced. The impacts of nonuniform spatial sampling in the virtual antenna array are analyzed, along with a multichannel filtering-based reconstruction algorithm. Conceptual system and discussions are provided. It is shown that high operation flexibility and reconfigurability can be obtained by utilizing the virtual antenna arrays provided by the MIMO SAR systems, thus enabling a satisfactory remote sensing performance.

\section{Introduction}

Multiple-input multiple-output radar has received much attention in recent years [1-3]; however, little work about MIMO synthetic aperture radar (SAR) has been investigated [4-6]. Note that the MIMO SAR discussed in this paper is different from the general MIMO radars in that aperture synthesis is employed in the MIMO SAR, but no aperture synthesis is employed in general MIMO radars [7]. Although SAR is a well-proven remote sensing application which obtains its high range resolution by utilizing the transmitted wide-band waveform and high azimuth resolution by exploiting the relative motion between the imaged target and the radar platform, current single-antenna SARs cannot provide some specific remote sensing performance, for example, simultaneously high-resolution and wide-swath (the width of the ground area covered by the radar beam) imaging $[8,9]$. MIMO SAR provides a solution to resolving these problems.
MIMO ideas are not new, their origin in control systems can be traced back to 1970s [10]. The early 1990s saw an emergence of MIMO ideas into the field of communication systems. More recently, the ideas of MIMO appears in sensor and radar systems. Given that MIMO SAR is in its infancy, there is no clear definition of what it is. It is generally assumed that independent signals are transmitted through different antennas, and these signals, after propagating through the environment, are received by multiple antennas. Unlike conventional phased array radars [11], in MIMO SARs each antenna transmits a unique waveform, orthogonal to the waveforms transmitted by other antennas. In the MIMO SAR receiver, a matched filter-bank is used to extract the orthogonal waveform components. When the orthogonal signals are transmitted from different antennas, the returns of each orthogonal signal will carry independent information about the remote sensing targets. The phase difference caused by different transmitting antennas along with the phase differences caused by different receiving 


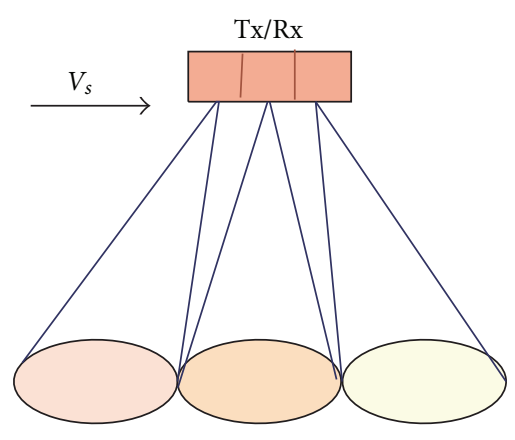

(a) single-phase centre multibeam

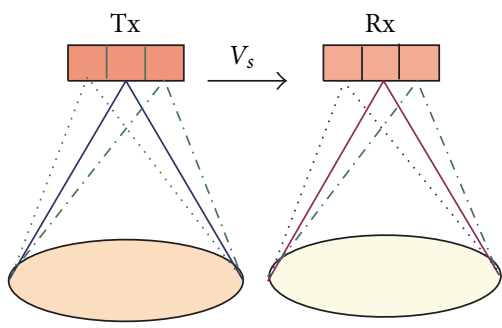

(b) multiple-phase centre multibeam

Figure 1: Geometry mode of the MIMO SAR antennas.

antennas can form a new virtual antenna array steering vector. With optimally designed antenna array positions, we can create a very long array steering vector with a small number of antennas [12-14]. More importantly, this provides high flexibility and reconfigurability in antenna configuration, thus enabling a flexible and reconfigurable SAR remote sensing performance.

Most of the MIMO SAR research so far focused on signal/ data models and corresponding signal processing algorithm [15-17]. Little work related to MIMO radar antenna can be found. The antenna effects on a monostatic MIMO radar for direction estimation were studied in [18] by analyzing the Cramer-Rao low bound (CRLB). Two different uniform linear antenna arrays, one narrowband and the another wideband, were investigated by exploring the CRLB. An iterative algorithm was proposed in [19] to design sparse MIMO radar transmit arrays to approximate a desired transmit beampattern response. Additionally, several minimum redundancy MIMO radars were proposed by other authors $[14,20-22]$. In fact, one of the main advantages of MIMO SAR is that the degrees of freedom can be greatly increased by the concept of virtual array provided by the multiple antennas. In this paper, we analyze the virtual antenna array design for MIMO SAR high-resolution wide-swath remote sensing, which has not been investigated in the literature.

The remaining sections are organized as follows. The system principle of the MIMO SAR is described in Section 2. The one-dimensional uniform and nonuniform linear antenna arrays are designed in Section 3. Their application potentials in high-resolution wide-swath remote sensing are also introduced. Next, Section 4 analyzes the impacts of nonuniform spatial sampling in the virtual antenna arrays. Finally, conceptual design system and discussions are provided in Section 5. This paper is concluded in Section 6.

\section{MIMO SAR Virtual Antenna Array}

There are two kinds of MIMO SAR configuration, as shown in Figure 1. The operation mode of the single phase centre multibeam (SPCM) MIMO SAR system is shown in Figure 1(a). A distinct channel is associated with each of the receive beams, and, hence, the data are split according to azimuth angular position or, equivalently, instantaneous
Doppler frequency centre in the azimuth direction. As a result, given knowledge of the relative squint angles of each beam (hence the Doppler center frequency for each beam) and assuming suitable isolation between the beams, each channel can be sampled at a Nyquist rate appropriate to the bandwidth covered by each narrow beam, instead of that covered by the full beamwidth. This arrangement enables correct sampling of the azimuth spectrum with a pulse repetition frequency (PRF) fitting the total antenna azimuth length, which is significantly smaller than the general PRF requirement.

The multiple phase centre multibeam (MPCM) MIMO SAR system also synthesizes multiple receive beams in the azimuth direction, as shown in Figure 1(b); however, the operating mode of this system is quite different from that of the previous one. In this case, the system transmits multiple broad beams and receives the radar returns in multiple beams which are displaced in the along-track direction. The motivation is that multiple independent sets of target returns are obtained for each transmitted pulse if the distance between phase centres is suitably set. This method basically implies that we may broaden the azimuth beam from the diffraction-limited width, giving rise to improved resolution, without having to increase the system operating PRF.

As noted previously, one of the main advantages of MIMO SAR is that the degrees of freedom can be greatly increased by the concept of virtual array [23]. Figure 2 illustrates a MIMO SAR system. Consider the MIMO SAR system with a transmit array equipped with $M$ colocated antennas and a receive array equipped with $N$ colocated antennas. Suppose both the transmit and receive arrays are close to each other in space (possibly the same array) so that they see targets at same directions.

The MIMO SAR received signal at each receiving antenna is the weighted summation of all the transmitted waveform

$$
\begin{array}{r}
r_{n}(t)=\sum_{m=1}^{M} a_{n, m} s_{m}(t), \quad m \in[1,2, \ldots, M], \\
n \in[1,2, \ldots, N],
\end{array}
$$

where $r_{n}(t)$ is the received signal at the $n$th antenna, $s_{m}(t)$ is the transmitted waveform at the $m$ th antenna, and $a_{n, m}$ is the channel coefficient with the $m$ th antenna as input and the 

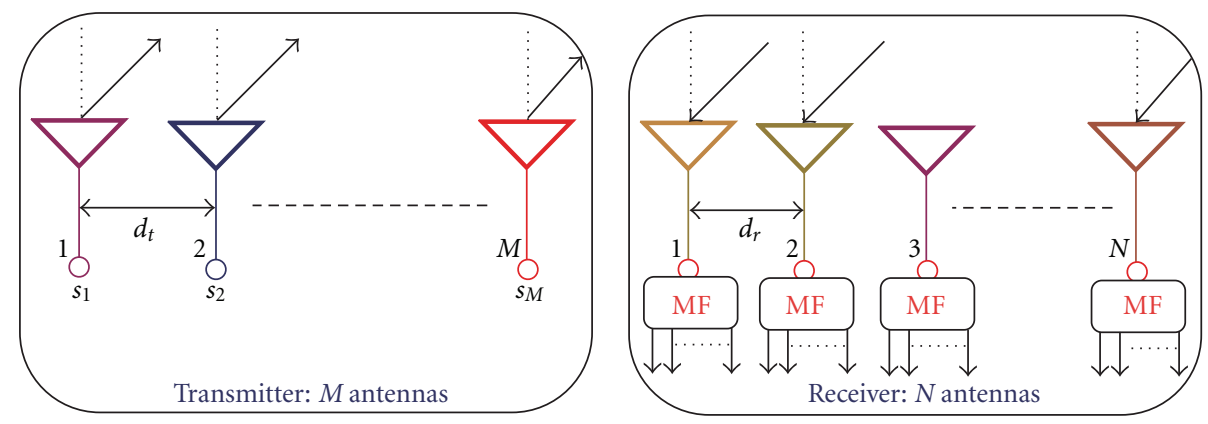

FIgURE 2: Illustration of an example MIMO SAR system.

$n$th antenna as output. When the transmitted waveforms are designed to be orthogonal

$$
\int s_{m}(t) s_{m^{\prime}}^{*}(t) \mathrm{d} t= \begin{cases}\delta(t), & m=m^{\prime} \\ 0, & m \neq m^{\prime}\end{cases}
$$

where ()$^{*}$ denotes a conjugate operator. At each receiving antenna, these orthogonal waveforms can then be extracted by $M$ matched filters. There are a total of $M \times N$ extracted signals. Compared to the traditional phased-array SAR where the same waveform is used at all the transmitting antennas and a total of $N$ coefficients are obtained for the matched filtering, the MIMO SAR gives more coefficients and, therefore, provides more degrees of freedom.

Suppose there are $K$ point targets, the received MIMO SAR signals can be written in a vector form

$$
\mathbf{x}(t)=\sum_{k=1}^{K} \sigma_{k}\left(\mathbf{a}^{T}\left(\theta_{k}\right) \mathbf{s}(t)\right) \mathbf{b}\left(\theta_{k}\right)+\mathbf{n}(t)
$$

where $\theta_{k}$ is the target direction, $\sigma_{k}$ is the complex-valued reflection coefficient of the focal point $\theta_{k}$ for the $k$ th point target, ()$^{T}$ is a transpose operator, $\mathbf{n}(t)$ is the noise vector, $\mathbf{a}\left(\theta_{k}\right)$ and $\mathbf{b}\left(\theta_{k}\right)$ are the actual transmit and actual receive steering vectors associated with the direction $\theta_{k}$. Without loss of generality, we ignore the noise in the following discussions. The SAR returns due to the $m$ th transmitted waveform can be extracted by matched filtering the received signal to each of the waveforms $s_{m}(t)$

$$
\mathbf{x}_{m}=\int \mathbf{x}(t) \mathbf{s}^{*}(t) \mathrm{d} t
$$

The $M N \times 1$ virtual target signal vector can then be written as

$$
\mathbf{y}=\sigma_{s} \mathbf{a}\left(\theta_{s}\right) \otimes \mathbf{b}\left(\theta_{s}\right)
$$

where $\otimes$ and $\theta_{s}$ denote the Kronker product and the target direction, respectively. Note that here perfect waveform orthogonality is assumed. This equation can be represented by

$$
\mathbf{y}=\sigma_{s} \mathbf{v}\left(\theta_{s}\right)
$$

where

$$
\mathbf{v}\left(\theta_{s}\right)=\mathbf{a}\left(\theta_{s}\right) \otimes \mathbf{b}\left(\theta_{s}\right)
$$

is the $M N \times 1$ steering vector associated with an virtual array of $M N$ sensors.

Suppose the transmitter has $M$ antennas, whereas the receiver has $N$ antennas, (7) means that a virtual antenna array with utmost number of $M N$ nonoverlapped virtual transmitting/receiving elements can be obtained to take full advantages of the MIMO antenna array. Since different antenna array configurations have different spatial sampling characteristics and signal processing complexity, the MIMO SAR antenna array configuration should be optimally designed.

\section{Linear Virtual Antenna Array}

3.1. Signal Models. Consider a linear transmitting array with $M$ antenna elements and a linear receiving array with $N$ antenna elements. Without loss of any generality, suppose the transmitting and the receiving arrays are parallel and colocated. The $m$ th transmitting antenna is located at $x_{T, m}=$ $(\lambda / 2) u_{m}$ and the $n$th receiving antenna is located at $x_{R, n}=$ $(\lambda / 2) v_{n}$, where $\lambda$ is the wavelength. Consider a far-field point target, the transmitter and receiver steering vectors can be represented, respectively, by

$$
\begin{aligned}
\mathbf{a}\left(\theta_{s}\right) & =\left[e^{j u_{1} \pi \sin \theta_{s}}, e^{j u_{2} \pi \sin \theta_{s}}, \ldots, e^{j u_{M} \pi \sin \theta_{s}}\right]^{T}, \\
\mathbf{b}\left(\theta_{s}\right) & =\left[e^{j v_{1} \pi \sin \theta_{s}}, e^{j v_{2} \pi \sin \theta_{s}}, \ldots, e^{j v_{N} \pi \sin \theta_{s}}\right]^{T} .
\end{aligned}
$$

From (7), we can get

$\mathbf{v}\left(\theta_{s}\right)=\left[\begin{array}{cccc}e^{j\left(v_{1}+u_{1}\right) \pi \sin \theta_{s}} & e^{j\left(v_{1}+u_{2}\right) \pi \sin \theta_{s}} & \cdots & e^{j\left(v_{1}+u_{M}\right) \pi \sin \theta_{s}} \\ e^{j\left(v_{2}+u_{1}\right) \pi \sin \theta_{s}} & e^{j\left(v_{2}+u_{2}\right) \pi \sin \theta_{s}} & \cdots & e^{j\left(v_{2}+u_{M}\right) \pi \sin \theta_{s}} \\ \vdots & \vdots & \cdots & \vdots \\ e^{j\left(v_{N}+u_{1}\right) \pi \sin \theta_{s}} & e^{j\left(v_{N}+u_{2}\right) \pi \sin \theta_{s}} & \cdots & e^{j\left(v_{N}+u_{M}\right) \pi \sin \theta_{s}}\end{array}\right]$.

Note that the amplitude of the signal reflected by the target has been normalized to unity. That is, the target response in the $m$ th matched filtering output of the $n$th receiving antenna is expressed as

$$
v_{m, n}\left(\theta_{s}\right)=e^{j\left(v_{n}+u_{m}\right) \pi \sin \theta_{s}} .
$$




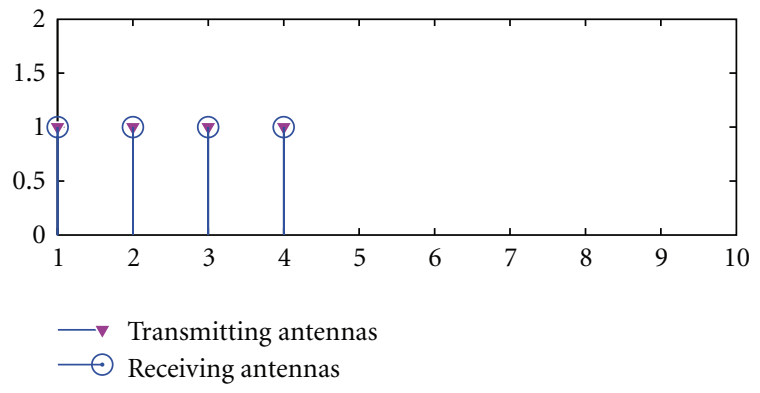

(a) transmitting and receiving antennas

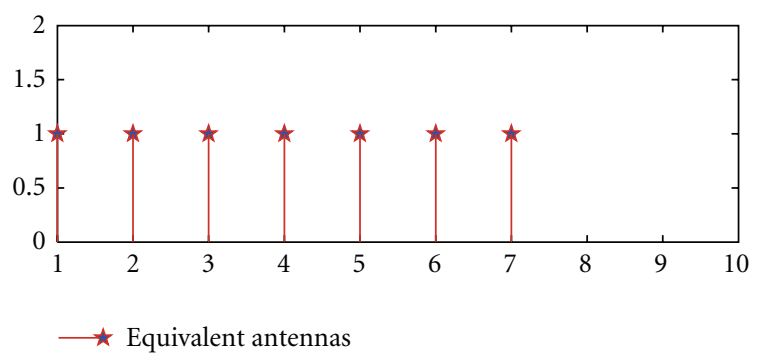

(b) equivalent virtual antennas

FigURE 3: Virtual phase centres of the uniform linear array.

It can be noticed that the phase differences are created by both the transmitting antenna locations and the receiving antenna locations. The target response expressed in (11) is the same as the target response received by a receiving array with $M N$ antenna elements located at

$$
\left\{x_{T, m}+x_{R, m}\right\}, \quad m \in[1,2, \ldots, M], n \in[1,2, \ldots, N] .
$$

The phase differences are created by both transmitting and receiving antenna locations. This $M N$-element array is just the virtual antenna array. An utmost number of $M N$-element virtual array can be obtained by using only $M+N$ physical antenna elements. It is as if we have a receiving array of $M N$ elements. The virtual antenna array can be seen as a way to sample the electromagnetic wave in the spatial domain. This degree-of-freedom can greatly increase the design flexibility of the MIMO SAR systems.

3.2. Effective Phase Centres. To investigate the effective phase centre caused by the virtual antenna array, in this section we consider several typical linear array configurations for MIMO SAR systems.

3.2.1. Transmitter Is Same to Receiver: $M=N=L$. If the transmitting array and the receiving array are uniform linear arrays, we assume that the first element of $\mathbf{a}\left(\theta_{s}\right)$ and $\mathbf{b}\left(\theta_{s}\right)$, respectively, is the reference element. From (8) and (9), we have

$$
\mathbf{a}\left(\theta_{s}\right)=\mathbf{b}\left(\theta_{s}\right)=\left[1, e^{j \pi \sin \theta_{s}}, e^{j 2 \pi \sin \theta_{s}}, \ldots, e^{j(M-1) \pi \sin \theta_{s}}\right]^{T} .
$$

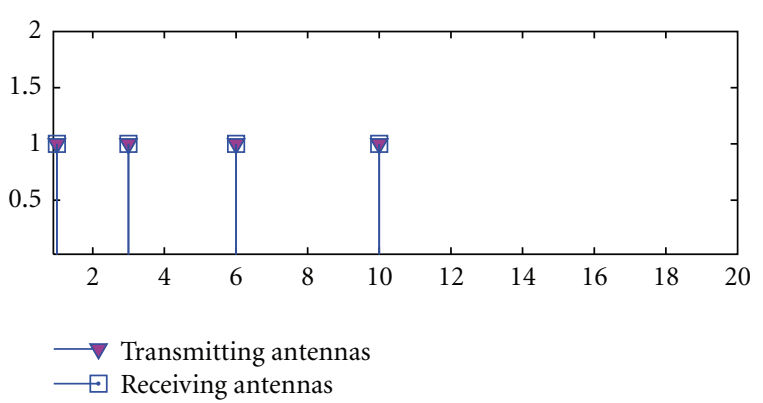

(a) transmitting and receiving antennas

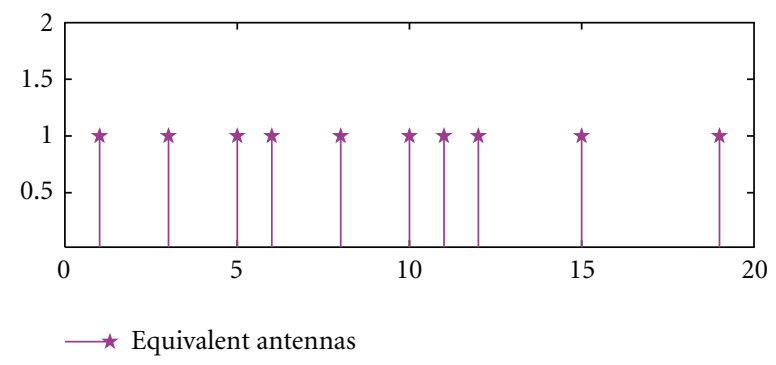

(b) equivalent virtual antennas

FIGURE 4: Virtual phase centres of the nonuniform linear array.

The equation (11) can then be reexpressed as

$$
\mathbf{v}\left(\theta_{s}\right)=\left[\begin{array}{cccc}
1 & e^{j \pi \sin \theta_{s}} & \cdots & e^{j(L-1) \pi \sin \theta_{s}} \\
e^{j \pi \sin \theta_{s}} & e^{j 2 \pi \sin \theta_{s}} & \cdots & e^{j L \pi \sin \theta_{s}} \\
\vdots & \vdots & \cdots & \vdots \\
e^{j(L-1) \pi \sin \theta_{s}} & e^{j M \pi \sin \theta_{s}} & \cdots & e^{j(2 L-3) \pi \sin \theta_{s}}
\end{array}\right] .
$$

In this case, the number of effective virtual phase centres is $2 L-1$ with the biggest virtual aperture of $2 L-2$. Suppose $M=N=4$, Figure 3 shows the corresponding virtual arrays.

If the transmitting array and the receiving array are nonuniform linear array, we can express the steering vector as

$$
\mathbf{a}\left(\theta_{s}\right)=\mathbf{b}\left(\theta_{s}\right)=\left[e^{j u_{1} \pi \sin \theta_{s}}, e^{j u_{2} \pi \sin \theta_{s}}, \ldots, e^{j u_{M} \pi \sin \theta_{s}}\right]^{T} .
$$

In this case, (11) can then be reexpressed as

$\mathbf{v}\left(\theta_{s}\right)=\left[\begin{array}{cccc}e^{j\left(2 u_{1}\right) \pi \sin \theta_{s}} & e^{j\left(u_{1}+u_{2}\right) \pi \sin \theta_{s}} & \cdots & e^{j\left(u_{1}+u_{M}\right) \pi \sin \theta_{s}} \\ e^{j\left(u_{2}+u_{1}\right) \pi \sin \theta_{s}} & e^{j\left(u_{2}+u_{2}\right) \pi \sin \theta_{s}} & \cdots & e^{j\left(u_{2}+u_{M}\right) \pi \sin \theta_{s}} \\ \vdots & \vdots & \cdots & \vdots \\ e^{j\left(u_{M}+u_{1}\right) \pi \sin \theta_{s}} & e^{j\left(u_{M}+u_{2}\right) \pi \sin \theta_{s}} & \cdots & e^{j\left(2 u_{M}\right) \pi \sin \theta_{s}}\end{array}\right]$.

It can be proved that the utmost number of effective virtual phase centres is $L(L+1) / 2$. Suppose also $M=N=4$; Figure 4 shows the corresponding virtual arrays.

\subsubsection{Transmitter and Receiver Have No Overlapped Elements.}

Suppose $M+N=L$; the utmost number of effective virtual phase centres can be determined by $L_{v}=N(L-N) \leq L^{2} / 4$. For $M=3, N=4$, Figure 5 shows two typical virtual arrays, one is uniform linear array and the other is nonuniform linear array. 


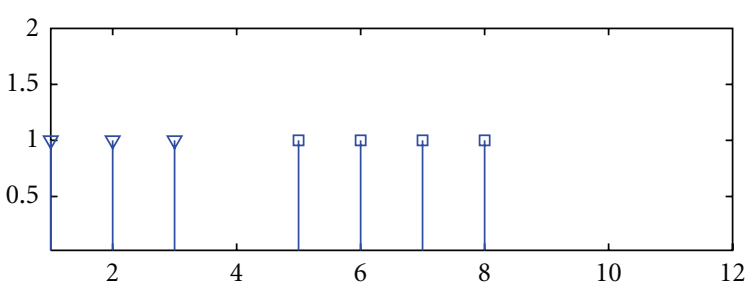

(a)

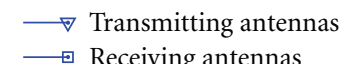

$\longrightarrow$ Receiving antennas

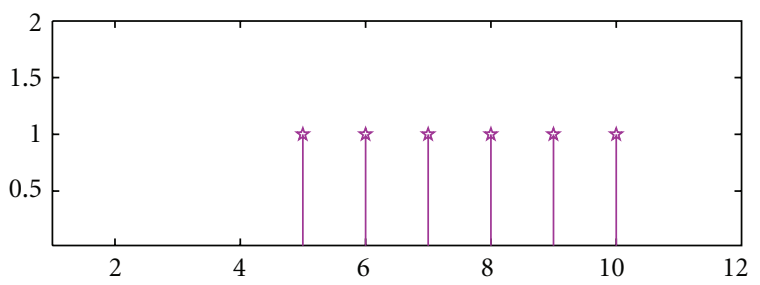

(b)

* Equivalent virtual antennas

(A) Uniform transmitting and/or receiving array

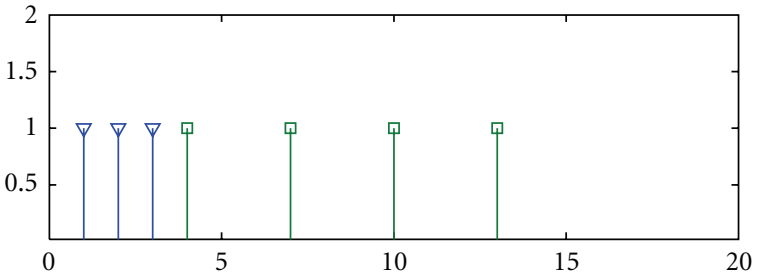

(a)
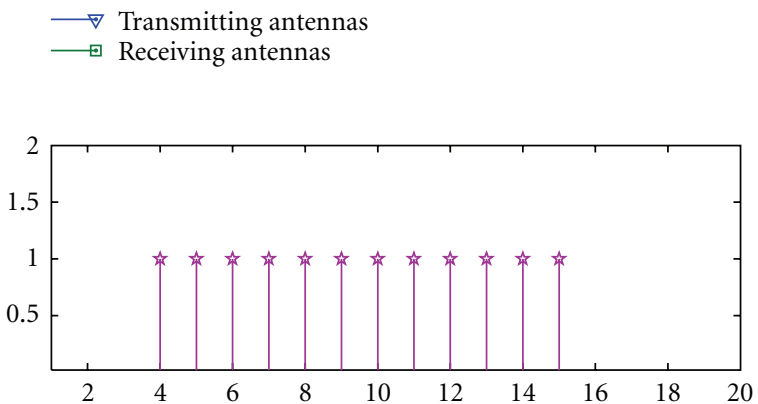

(b)

Ł Equivalent virtual antennas

(B) Nonuniform transmitting and/or receiving array

Figure 5: The azimuth-variant Doppler characteristics: Case A: using spaceborne transmitter, Case B: using airborne transmitter.

3.2.3. Transmitter and Receiver Have Overlapped Elements. Suppose the transmitter and receiver have $N_{o v}$ overlapped elements, the utmost number of effective virtual phase centres is determined by

$$
L_{v} \leq \frac{N_{o v}\left(N_{o v}+1\right)}{2}+\left(M-N_{o v}\right)\left(N-N_{o v}\right) .
$$

Suppose also $M=4, N=3$; Figure 6 shows the corresponding virtual arrays.

Comparing the three cases discussed above, we can concluded that the minimum redundant array is obtained when the transmitting array and/or the receiving array are nonuniform linear array.

3.3. System Performance Analysis. Future SAR will be required to produce high-resolution imagery over a wide area of surveillance. However, the minimum antenna area constraint makes it a contradiction to simultaneously obtain both unambiguous high azimuth resolution and wide-swath. As well as consideration of antenna beam-width, the actual achievable resolution and swath for a SAR is subject to a number of restrictions imposed by various operating factors. The details can be found in $[24,25]$. A basic limitation is the minimum antenna area constraint, which can be represented by

$$
A_{a} \geq \frac{4 v_{s} \lambda R_{c} \tan \eta}{c_{0}}
$$

where $v_{s}$ is the velocity of SAR platform, $R_{c}$ is the slant range from radar to mid-swath, $\eta$ is the incidence angle, and $c_{0}$
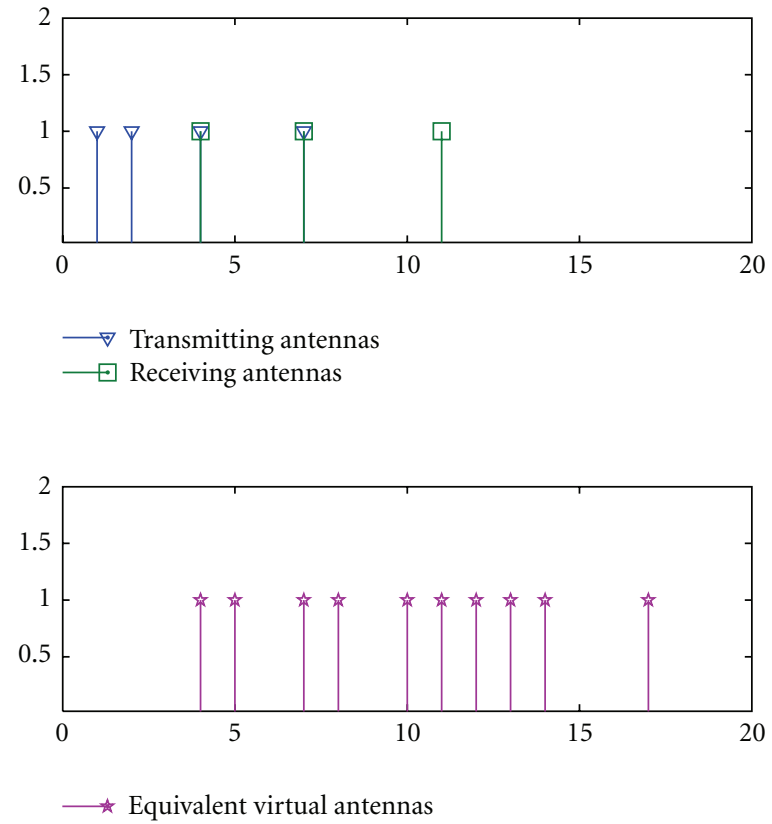

FIGURE 6: Virtual phase centres of the nonuniform linear array.

is the speed of light. This requirement arises because the illuminated area of the ground must be restricted so that the radar does not receive ambiguous returns in range or/and Doppler. In this respect, a high operating PRF is desired for suppressing azimuth ambiguity. But the magnitude of the operating PRF is limited by the range ambiguity requirement. 


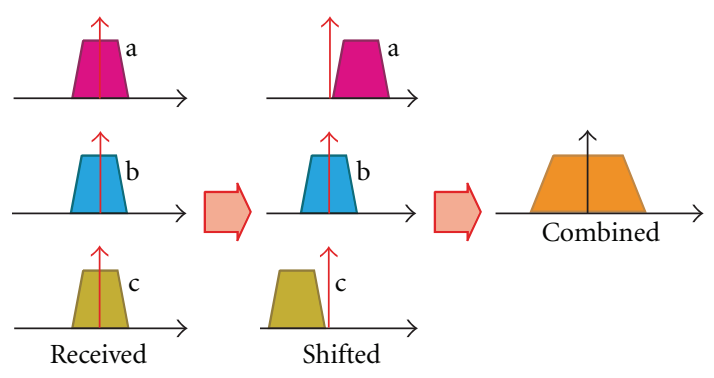

Figure 7: Azimuthal spectra synthesis for multichannel subsampling. Here three channels are assumed. It is for illustration only.

The attainment of wide-swath will become increasingly difficult if higher spatial resolution is required, due to the requirement of increased PRF. The MIMO SAR can overcome the minimum antenna area. The virtual effective phase centres enable correct sampling of the azimuth spectrum with a PRF fitting the total antenna azimuth length, which is $L_{v}$ times smaller than the general PRF requirement. Correspondingly, the area of each antenna is restricted by

$$
A_{a} \geq \frac{4 v_{s} \lambda R_{c} \tan \eta}{c_{0}} \cdot \frac{1}{L_{v}} .
$$

Clearly the minimum antenna area is $L_{v}$-times smaller than the respective area of a monostatic SAR. Thereafter, the displaced phase center antenna (DPCA) technique [26, 27] can be used to gain additional samples along the synthetic aperture which enables an efficient suppression of azimuth ambiguities, that is, the multiple beams in azimuth allow for the division of a broad Doppler spectrum into multiple narrow-band subspectra with different Doppler centroids. A coherent combination of the subspectra will then yield a broad Doppler spectrum for high azimuth resolution, as shown in Figure 7 [28]. Thus this approach is especially attractive for high-resolution SAR imaging that uses a long antenna for unambiguous wide-swath remote sensing.

For a given range and azimuth antenna pattern, the PRF must be selected such that the total ambiguity noise contribution is enough small relative to the signal. Alternately, given a PRF or range of PRFs, the antenna dimensions should be enough small such that the ambiguity-to-noise ratio specification is met. Thus, the MIMO SAR system performance can be evaluated by the azimuth ambiguity to signal ratio (AASR), which is defined as [29]

$$
A A S R \approx \frac{\sum_{m=-\infty, m \neq 0}^{\infty} \int_{-0.5 B_{d}}^{0.5 B_{d}} G^{2}(f+m \cdot \mathrm{PRF}) \mathrm{d} f}{\int_{-0.5 B_{d}}^{0.5 B_{d}} G^{2}(f) \mathrm{d} f},
$$

where $B_{d}$ is the SAR correlator azimuth processing bandwidth, $G(f)$ is the equivalent azimuth transmit-receive antenna pattern, and PRF is the value of PRF.

As an example, we consider only the MIMO SAR configuration in which the transmitter is same to the receiver, for example, the configuration illustrated in Figure 3. Suppose the transmitting antennas synchronize perfectly with the receiving antennas, the $k$ th antenna beam can be represented by

$$
G_{k}(\theta)=\sin c^{2}\left(\frac{\pi L_{a s} \cos \left(k \cdot \theta_{a}\right)}{\lambda} \sin \left(\theta-k \cdot \theta_{a}\right)\right),
$$

where $L_{a s}$ is the subantenna length, $\theta$ is the antenna beamwidth in elevation, and $\theta_{a}$ is the antenna beam-width in azimuth. Note that here the central antenna element is assumed as the reference element. As the $3 \mathrm{~dB}$ beam-width can be approximately determined by

$$
\theta \approx \frac{\lambda}{2 v_{s}} f, \quad \theta_{a}=k_{a} \frac{\lambda}{L_{a s}}
$$

with $k_{a}$ a given constant, we can get

$$
\begin{aligned}
& \operatorname{AASR}_{k}(P R F) \\
& =\left\{\sum _ { m = - \infty m \neq 0 } ^ { \infty } \left[\int_{(i-0.5) B_{d s}}^{(i+0.5) B_{d s}} G_{k}^{2}(f+m \cdot \mathrm{PRF}) \mathrm{d} f\right.\right. \\
& +\sum_{j \neq k} \int_{(i-0.5) B_{d s}}^{(i+0.5) B_{d s}} G_{k}(f+m \cdot P R F) G_{j} \\
& \times(f+m \cdot \mathrm{PRF}) \mathrm{d} f]\} \\
& \quad\left\{\int_{(i-0.5) B_{d s}}^{(i+0.5) B_{d s}} G_{k}^{2}(f) \mathrm{d} f+\sum_{j \neq k} \int_{(i-0.5) B_{d s}}^{(i+0.5) B_{d s}} G_{k}(f) G_{j}(f) \mathrm{d} f\right\}^{-1},
\end{aligned}
$$

where $B_{d s}$ is the Doppler bandwidth of each subantenna.

\section{Impacts of Nonuniform Spatial Sampling}

As investigated previously, different array configurations have different spatial sampling characteristics and signal processing complexity. A uniform array is desired, so that the complexity of signal processing can be reduced, and the ultimate MIMO SAR image quality can be ensured. To reach this aim, the optimum PRF must be satisfactory with

$$
\mathrm{PRF}_{\text {uni }}=\frac{2 v_{s}}{L_{v} d_{a}}
$$

where $d_{a}$ is azimuth separation between the virtual array elements. This imposes a stringent requirement on the system as it states that to ensure equal spacing between all samples in azimuth the PRF has to be chosen such that the SAR platform moves just one half of its antenna length between subsequent radar pulses. This optimum PRF yields a data array equivalent to that of a single-aperture system operating with $L_{v}$. PRF. In reverse, any deviation from the relation will result in a nonequally sampled data array along the synthetic aperture that is no longer equivalent to a monostatic signal and cannot be processed by conventional monostatic algorithms without performance degradation.

To analyze the impact of nonuniform displaced phase centre sampling, we consider the received radar returns

$$
s_{i}(t, \tau) \approx \sigma_{i}\left[h_{0}(t) *_{t} h_{1, i}(t, \tau)\right], \quad i=1,2, \ldots, N,
$$


where $t$ is the range fast time, $\tau$ is the azimuth slow time, and $*_{t}$ is a convolution operator on the variable $t . h_{0}(t)$ and $h_{1, i}(t, \tau)$ denote, respectively, the range reference function and azimuth reference function

$$
\begin{aligned}
h_{0}(t)= & w_{r}(t) \cdot \exp \left(-j \pi k_{r} t^{2}\right), \\
h_{1, i}(t, \tau)= & \exp \left\{-j \frac{2 \pi}{\lambda}\left[R_{c}(\tau)+R_{c}\left(\tau+i \frac{d_{a}}{v_{s}}\right)\right]\right\} \\
& \times w_{a}(\tau) \cdot \delta\left[\tau-\frac{R_{c}(\tau)+R_{c}\left(\tau+i d_{a} / v_{s}\right)}{c_{0}}\right],
\end{aligned}
$$

where $R_{c}(t)$ is the equivalent slant range and $k_{r}$ is the chirp rate of the transmitted waveforms. Note that perfect orthogonal frequency diversion multiplexing (OFDM) linearly frequency modulation (LFM) waveforms are assumed in this paper. $w_{r}(t)$ and $w_{a}(\tau)$ denote the antenna pattern in range dimension and azimuth dimension, respectively. Since

$$
R_{c}(\tau)+R_{c}\left(\tau+i \frac{d_{a}}{v_{s}}\right) \approx 2 R_{c}\left(\tau+i \frac{d_{a}}{2 v_{s}}\right),
$$

we then have

$$
s_{i}(t, \tau) \approx \sigma_{i}\left[h_{0}(t) \otimes_{t} h_{1}\left(t, \tau+i \frac{d_{a}}{2 v_{s}}\right)\right] .
$$

with

$$
\begin{aligned}
h_{1}\left(t, \tau+i \frac{d_{a}}{2 v_{s}}\right)= & w_{a}\left(\tau+i \frac{d_{a}}{2 v_{s}}\right) \cdot \exp \left\{-j \frac{4 \pi}{\lambda} R_{c}\left(\tau+i \frac{d_{a}}{2 v_{s}}\right)\right\} \\
& \cdot\left[\tau-\frac{2 R_{c}\left(\tau+i\left(d_{a} / 2 v_{s}\right)\right)}{c_{0}}\right] .
\end{aligned}
$$

Equivalently, the nonuniform PRF can be considered as azimuth time drift

$$
\tau_{e r}=\frac{d_{a}}{2 v_{s}}-\frac{1}{L_{v} \cdot \mathrm{PRF}} .
$$

After matched filtering and range mitigation correction, we can get

$$
\begin{aligned}
& s_{i}\left(k \frac{1}{L_{v} \cdot \mathrm{PRF}}\right) \\
& =w_{a}\left(k \frac{1}{L_{v} \cdot \mathrm{PRF}}+i \cdot \tau_{e r}\right) \\
& \quad \times \exp \left\{-j\left[2 \pi f_{d}\left(\frac{1}{L_{v} \cdot \mathrm{PRF}}+i \cdot \tau_{e r}\right)\right.\right. \\
& \left.\left.\quad+\pi k_{a}\left(k \frac{1}{L_{v} \cdot \mathrm{PRF}}+i \cdot \tau_{e r}\right)^{2}\right]\right\},
\end{aligned}
$$

where $k$ is an integer, $f_{d}$ is the Doppler frequency centroid, and $k_{a}$ is the Doppler chirp rate. It is noticed that the signals are periodic nonuniform with the period of 1/PRF. This information is particularly importantly for developing nonuniform reconstruction algorithms. The impacts of

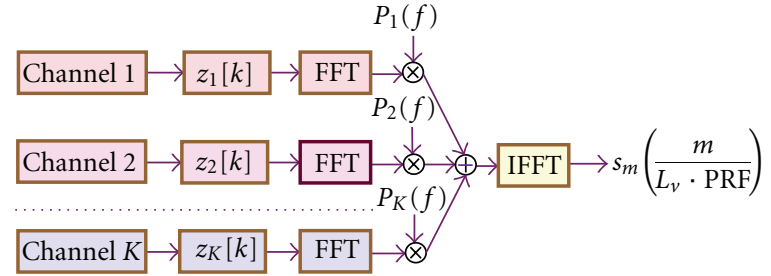

FIGURE 8: Reconstruction filtering for multichannel subsampling in case of three channels.

nonuniform spectral sampling can be evaluated by the following expression [8]:

$$
\begin{aligned}
s_{i}(\tau)=w_{a}(\tau) & \exp \left(-j \pi k_{a} \tau^{2}\right) \\
\times & \quad\left\{J_{0}\left(2 \pi f_{d} \tau_{e r} b_{n}\right)\right. \\
& -j 2 \pi k_{d} \tau_{e r} b_{n} J_{0}\left(2 \pi f_{d} \tau_{e r} b_{n}\right) \tau \sin \left(\frac{2 \pi \tau}{L_{v} \cdot \mathrm{PRF}}\right) \\
& \left.-2 j J_{1}\left(2 \pi f_{d} \tau_{e r} b_{n}\right) \sin \left(\frac{2 \pi \tau}{L_{v} \cdot \mathrm{PRF}}\right)\right\} .
\end{aligned}
$$

The ambiguous Doppler spectrum of a nonuniformly sampled SAR signal can be recovered unambiguously by applying a system of reconstruction filters. The algorithm illustrated in Figure 8 is based considering the data acquisition in the MIMO SAR as a linear system with multiple receiver channels, each is described by a linear filter. The reconstruction consists essentially of multiple linear filters which are individually applied to the subsampled signals of the receiver channels and then combined coherently. The details can be found in $[30,31]$.

Therefore, the optimal MIMO SAR configuration should have a uniform virtual linear array along the azimuth dimension. Consider a MIMO SAR with an $M$-element $p$ spaced transmitting uniform linear array and an $N$-element $q$-spaced receiving uniform linear array. According to the proposition discussed in [32]. The virtual array is an effective uniform linear array if and only if $1 \leq \gamma \leq N$ with the ratio coefficient $\gamma=p / q$ or $1 \leq \gamma_{0} \leq M$ with $\gamma_{0}=1 / \gamma$. Moreover, the virtual array is a nonoverlapped $M N$-element uniform linear array if and only if $\gamma=N$ or $\gamma=1 / M$.

\section{Conceptual System Design and Discussions}

To further evaluate the quantitative performance, an example MIMO SAR system is considered. The MIMO SAR operates in X-band with a center frequency of $10 \mathrm{GHz}$. The geometric ground-range and azimuth resolution are set to $\rho_{r}=$ $0.2 m$ and $\rho_{a}=0.2 m$, respectively. To calculate the system performance, an overall loss factor $L_{f}=3 \mathrm{~dB}$, a fixed flying height of $30 \mathrm{~km}$, and a receiver noise figure of $F=3 \mathrm{~dB}$ are assumed. It is further assumed that the signal bandwidth is adjusted for varying angle of incidence such that the groundrange resolution is constant across the swath. One example system design is provided in Table 1 . We can notice that, for the incidence angle given in Table 1 a swath of $18 \mathrm{~km}$ 


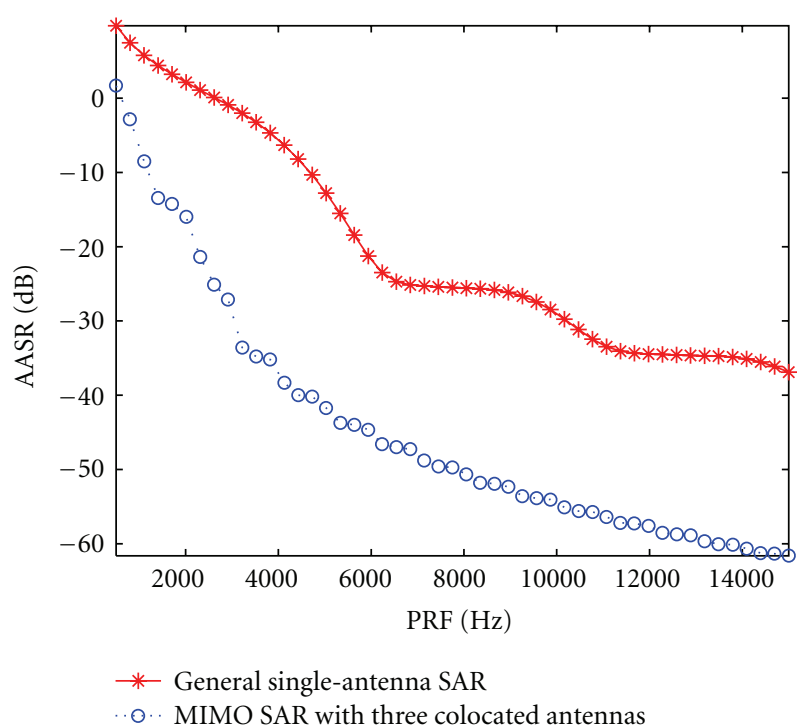

FIGURE 9: Comparative AASR results between conventional singleantenna SAR as a function of PRF.
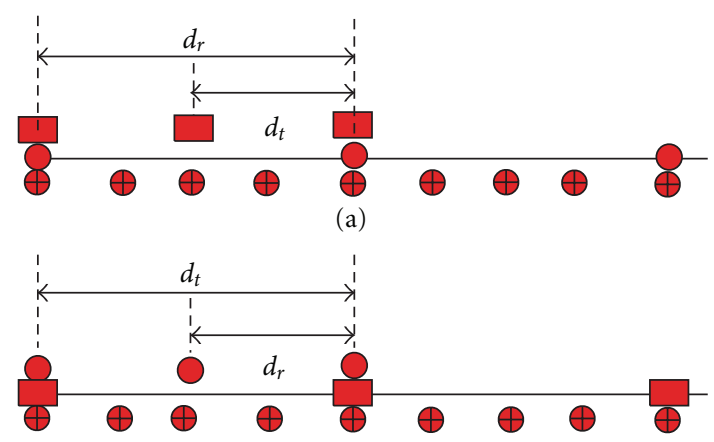

(b)

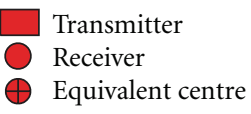

FIgUre 10: Two general linear antenna configurations for MIMO SAR systems.

and a noise equivalent sigma zero (NESZ) of $-48 \mathrm{~dB}$ can be obtained with a total antenna size not larger than that of current systems. Note that the NESZ is defined as the target radar cross section for which the final SAR image SNR is equal to one (i.e., $\mathrm{SNR}_{\text {image }}=0 \mathrm{~dB}$ ).

It is also worthwhile to compare its AASR performance to conventional single-aperture SAR. Consider again the system parameters listed in Table 1; Figure 9 gives the comparative AASR performance as a function of PRF. In SAR remote sensing applications, AASR is typically specified to be on the order of $-20 \mathrm{~dB}$, but a lower AASR is desired. It can be noticed from Figure 9 that the AASR is typically below $-20 \mathrm{~dB}$ with a low operating PRF requirement. This means that a wider swath can be obtained.

The equivalent virtual antenna pattern can be impacted by the different antenna configurations, even for the same number of equivalent virtual antenna array. Figure 10 shows

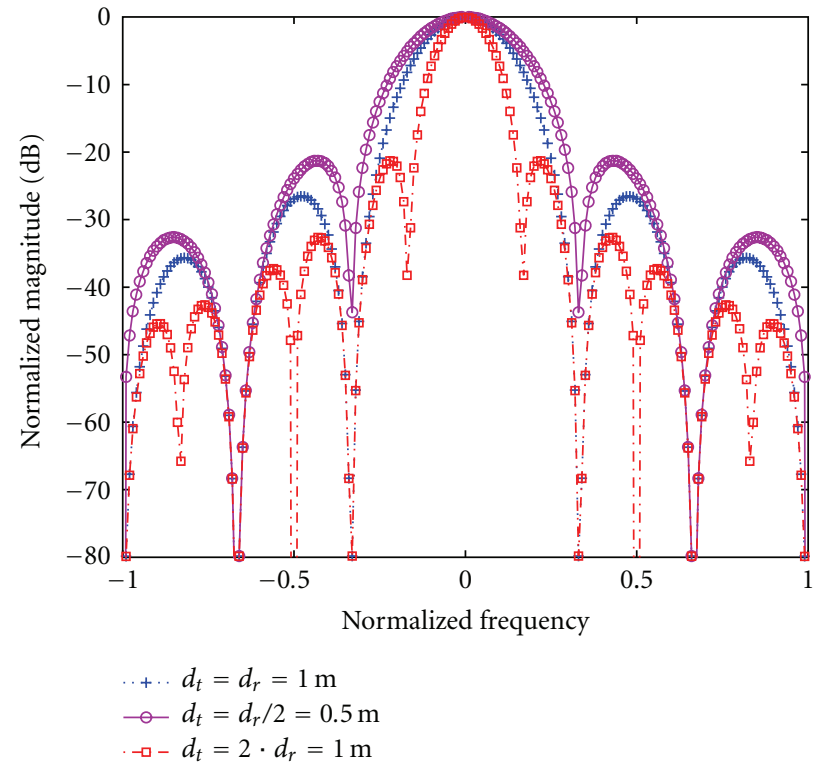

FIGURE 11: Equivalent antenna pattern of the MIMO SAR configurations.

TABle 1: Performance parameters of an example MIMO SAR system.

\begin{tabular}{lcc}
\hline Parameters & Variables & Values \\
\hline Mean transmit power & $P_{\text {avg }}$ & $10 \mathrm{~W}$ \\
Number of transmitting antennas & $M$ & 3 \\
Number of receiving antennas & $N$ & 3 \\
Transmit/receive antenna length & $L_{a s}$ & $0.9 \mathrm{~m}$ \\
Platform altitude & $h_{s}$ & $30 \mathrm{~km}$ \\
Platform velocity & $v_{s}$ & $500 \mathrm{~m} / \mathrm{s}$ \\
Incidence angle & $\eta$ & $30^{\circ}$ \\
$\quad$ Antenna width & $H_{a}$ & $0.1 \mathrm{~m}$ \\
$\quad$ Swath width & $W_{s}$ & $12 \mathrm{~km}$ \\
$\quad$ Radiometric resolution & $N E S Z$ & $-51.16 \mathrm{~dB}$ \\
Incidence angle & $\eta$ & $45^{\circ}$ \\
$\quad$ Antenna width & $H_{a}$ & $0.1 \mathrm{~m}$ \\
Swath width & $W_{s}$ & $18 \mathrm{~km}$ \\
$\quad$ Radiometric resolution & $N E S Z$ & $-48.52 \mathrm{~dB}$ \\
Incidence angle & $\eta$ & $45^{\circ}$ \\
$\quad$ Antenna width & $H_{a}$ & $0.2 \mathrm{~m}$ \\
Swath width & $W_{s}$ & $9 \mathrm{~km}$ \\
Radiometric resolution & $N E S Z$ & $-54.54 \mathrm{~dB}$ \\
\hline
\end{tabular}

two general linear antenna configurations, where $d_{t}$ and $d_{r}$ denote the distance separation between two neighboring antennas for the transmitter and the receiver, respectively. Figure 11 shows the comparative equivalent antenna patterns for the two antenna configurations. It can be noticed that different equivalent MIMO SAR antenna patterns can be obtained by choosing different array configurations. This provides a potential to develop new GMTI or remote sensing approaches. 


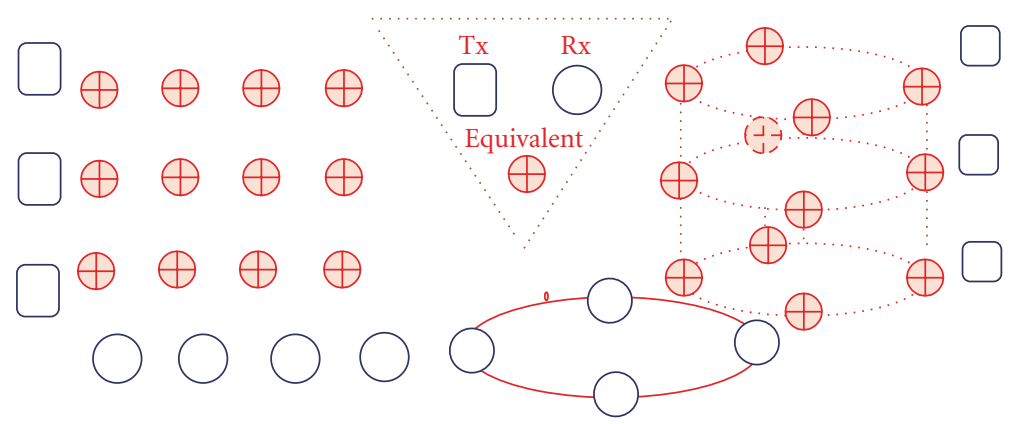

FIGURE 12: Example two-dimensional planar virtual array and three-dimensional cylindrical virtual array.

Another note is that, in this paper, we considered only the one-dimensional linear array. Two-dimensional and threedimensional phantom element array can also be synthesized midway between each transmitter-receiver pair. Figure 12 shows an example two-dimensional, planar array and a three-dimensional cylindrical array. Note that many other forms of two-dimensional three-dimensional arrays can be obtained in a similar way.

\section{Conclusion}

MIMO SAR is a recently proposed remote sensing concept. It has been shown that MIMO SAR can be used to improve remote sensing system performance. One of the main advantages of MIMO SAR is that the degrees of freedom can be greatly increased by the concept of virtual antenna array. In this paper, we investigated the virtual linear antenna array for MIMO SAR high-resolution wide-swath remote sensing applications. The impacts of nonuniform spatial sampling in the virtual antenna array are analyzed, along with a multichannel filtering-based reconstruction algorithm. The virtual high-dimensional antenna arrays are also investigated. Conceptual design system is provided, along with the system performance. It is shown that high operation flexibility and reconfigurability can be obtained by utilizing the virtual antenna arrays provided by the MIMO SAR systems, thus enabling a satisfactory remote sensing performance. High-dimensional virtual antenna arrays including two-dimensional planar array and threedimensional cylindrical array are also discussed, with an aim for further investigations. In a subsequent work, we plan to further investigate the MIMO SAR sparse antenna array design and the corresponding signal processing algorithms to resolve the spatial nonuniform sampling problems.

\section{Acknowledgments}

This work was supported in part by the National Natural Science Foundation of China under Grant no. 41101317, the Fundamental Research Funds for the Central Universities under Grant no. ZYGX2010J001, the First Grade of 49th Chinese Post-Doctor Research Funds under Grant no. 20110490143, and the Open Funds of the State Laboratory of Remote Sensing Science, Institute of Remote Sensing
Applications, Chinese Academy of Sciences under Grant no. OFSLRSS201011.

\section{References}

[1] J. Li and P. Stoica, MIMO Radar Signal Processing, John Wiley \& Sons, New York, NY, USA, 2008.

[2] J. Li and P. Stoica, "MIMO radar with colocated antennas," IEEE Signal Processing Magazine, vol. 24, no. 5, pp. 106-114, 2007.

[3] A. M. Haimovich, R. S. Blum, and L. J. Cimini, "MIMO radar with widely separated antennas," IEEE Signal Processing Magazine, vol. 25, no. 1, pp. 116-129, 2008.

[4] X. Zhuge and A. G. Yarovoy, "A sparse aperture MIMO-SARbased UWB imaging system for concealed weapon detection," IEEE Transactions on Geoscience and Remote Sensing, vol. 49, no. 1, pp. 509-518, 2011.

[5] D. Cristallini, D. Pastina, and P. Lombardo, "Exploiting MIMO SAR potentialities with efficient cross-track constellation configurations for improved range resolution," IEEE Transactions on Geoscience and Remote Sensing, vol. 49, no. 1, pp. 38-52, 2011.

[6] W.-Q. Wang, "Space-time coding MIMO-OFDM SAR for high-resolution imaging," IEEE Transactions on Geoscience and Remote Sensing, vol. 49, no. 8, pp. 3094-3104, 2011.

[7] W.-Q. Wang, "Applications of MIMO technique for aerospace remote sensing," in Proceedings of Aerospace Remote Sensing, Big Sky, Mont, USA, March 2007.

[8] W.-Q. Wang, Multi-Antenna Synthetic Aperture Radar Imaging Theory and Practice, National Defense Industry Press, Beijing, China, 2010.

[9] W.-Q. Wang, Near-Space Remote Sensing: Potential and Challenges, Springer, Berlin, Germany, 2011.

[10] R. K. Mehra, "Optimal inout signals for parameter estimation in dynamic systems - survey and new results," IEEE Transactions on Automatic Control, vol. 19, no. 6, pp. 753-768, 1974.

[11] M. Bachmann, M. Schwerdt, and B. Bräutigam, "Accurate antenna pattern modeling for phased array antennas in SAR applications-demonstration on TerraSAR-X," International Journal of Antennas and Propagation, vol. 2009, Article ID 492505, 9 pages, 2009.

[12] D. R. Fuhrmann, J. P. Browning, and M. Rangaswamy, "Signaling strategies for the hybrid MIMO phased-array radar," IEEE Journal on Selected Topics in Signal Processing, vol. 4, no. 1, pp. 66-78, 2010.

[13] A. Hassanien and S. A. Vorobyov, "Phased-mimo radar: a tradeoff between phased-array and mimo radars," IEEE 
Transactions on Signal Processing, vol. 58, no. 6, pp. 3137-3151, 2010.

[14] C. Y. Chen and P. P. Vaidyanathan, "Minimum redundancy MIMO radars," in Proceedings of IEEE International Symposium on Circuits and Systems (ISCAS '08), pp. 45-48, Seattle, Wash, USA, May 2008.

[15] Y. Lin, B. Zhang, W. Hong, Y. Wu, and Y. Li, "MIMO SAR processing with azimuth nonuniform sampling," in Proceedings of IEEE International Geoscience and Remote Sensing Symposium (IGARSS '10), pp. 4652-4655, Honolulu, Hawaii, USA, 2010.

[16] W.-Q. Wang, Q. Peng, and J. Cai, "Diversified MIMO SAR waveform analysis and generation," in Proceedings of the 2 nd IEEE Asian-Pacific Conference on Synthetic Aperture Radar (APSAR '09), pp. 270-273, Xi' an, China, October 2009.

[17] V. K. Ithapu, A. K. Mishra, and R. K. Panigrahi, "Diversity employment into target plus clutter SAR imaging using MIMO configuration," in Proceedings of Indian Antenna Week on Advanced Antenna Technology (AAT '10), pp. 1-4, Puri, India, June 2010.

[18] X. H. Wu, A. A. Kishk, and A. W. Glisson, "Antenna effects on a monostatic MIMO radar for direction estimation, a CramèrRao lower bound analysis," IEEE Transactions on Antennas and Propagation, vol. 59, no. 6, pp. 2388-2395, 2011.

[19] W. Roberts, L. Xu, J. Li, and P. Stoica, "Sparse antenna array design for MIMO active sensing applications," IEEE Transactions on Antennas and Propagation, vol. 59, no. 3, pp. 846-858, 2011.

[20] J. Dong, Q. Li, and W. Guo, "A combinatorial method for antenna array design in minimum redundancy MIMO radars," IEEE Antennas and Wireless Propagation Letters, vol. 8, pp. 1150-1153, 2009.

[21] G. Liao, M. Jin, and J. Li, "A two-step approach to construct minimum redundancy MIMO radars," in Proceedings of International Radar Conference-Surveillance for a Safer World, pp. 1-4, Bordeaux, France, 2009.

[22] M. Jin, G. Liao, J. Li, and W. Li, "Direction finding using minimum redundancy MIMO radar," in Proceedings of IET International Radar Conference, pp. 1-4, Guilin, China, 2009.

[23] D. W. Bliss and K. W. Forsythe, "Multiple-input multipleoutput (MIMO) radar and imaging: degrees of freedom and resolution," in Proceedings of the 37th IEEE Asilomar Conference on Signals, Systems, and Computers, pp. 54-59, Pacific Grove, Calif, USA, November 2003.

[24] A. Currie and M. A. Brown, "Wide-swath SAR," IEE Proceedings, Part F, vol. 139, no. 2, pp. 122-135, 1992.

[25] P. Capece, "Active SAR antennas: design, development, and current programs," International Journal of Antennas and Propagation, vol. 2009, Article ID 796064, 11 pages, 2009.

[26] A. Bellettini and M. A. Pinto, "Theoretical accuracy of synthetic aperture sonar micronavigation using a displaced phase-center antenna," IEEE Journal of Oceanic Engineering, vol. 27, no. 4, pp. 780-789, 2002.

[27] P. Lombardo, F. Colone, and D. Pastina, "Monitoring and surveillance potentialities obtained by splitting the antenna of the COSMO-SkyMed SAR into multiple sub-apertures," IEE Proceedings: Radar, Sonar and Navigation, vol. 153, no. 2, pp. 104-116, 2006.

[28] W.-Q. Wang, "Near-space wide-swath radar imaging with multiaperture antenna," IEEE Antennas and Wireless Propagation Letters, vol. 8, pp. 461-464, 2009.

[29] F. K. Li and W. T. K. Johnson, "Ambiguities in spaceborne synthetic aperture radar systems," IEEE Transactions on Aerospace and Electronic Systems, vol. 19, no. 3, pp. 389-397, 1983.
[30] N. Gebert, G. Krieger, and A. Moreira, "SAR signal reconstruction from non-uniform displaced phase centre sampling in the presence of perturbations," in Proceedings of IEEE Geoscience and Remote Sensing Symposium, pp. 1034-1037, Seoul, Korea, 2005.

[31] G. Krieger, N. Gebert, and A. Moreira, "Unambiguous SAR signal reconstruction from nonuniform displaced phase center sampling," IEEE Geoscience and Remote Sensing Letters, vol. 1, no. 4, pp. 260-264, 2004.

[32] L. Wang, J. Xu, S. Peng, J. Yuan, and X. Ma, "Optimal linear array configuration and DOF tradeoff for MIMO-SAR," Chinese Journal of Electronics, vol. 20, no. 2, pp. 380-384, 2011. 

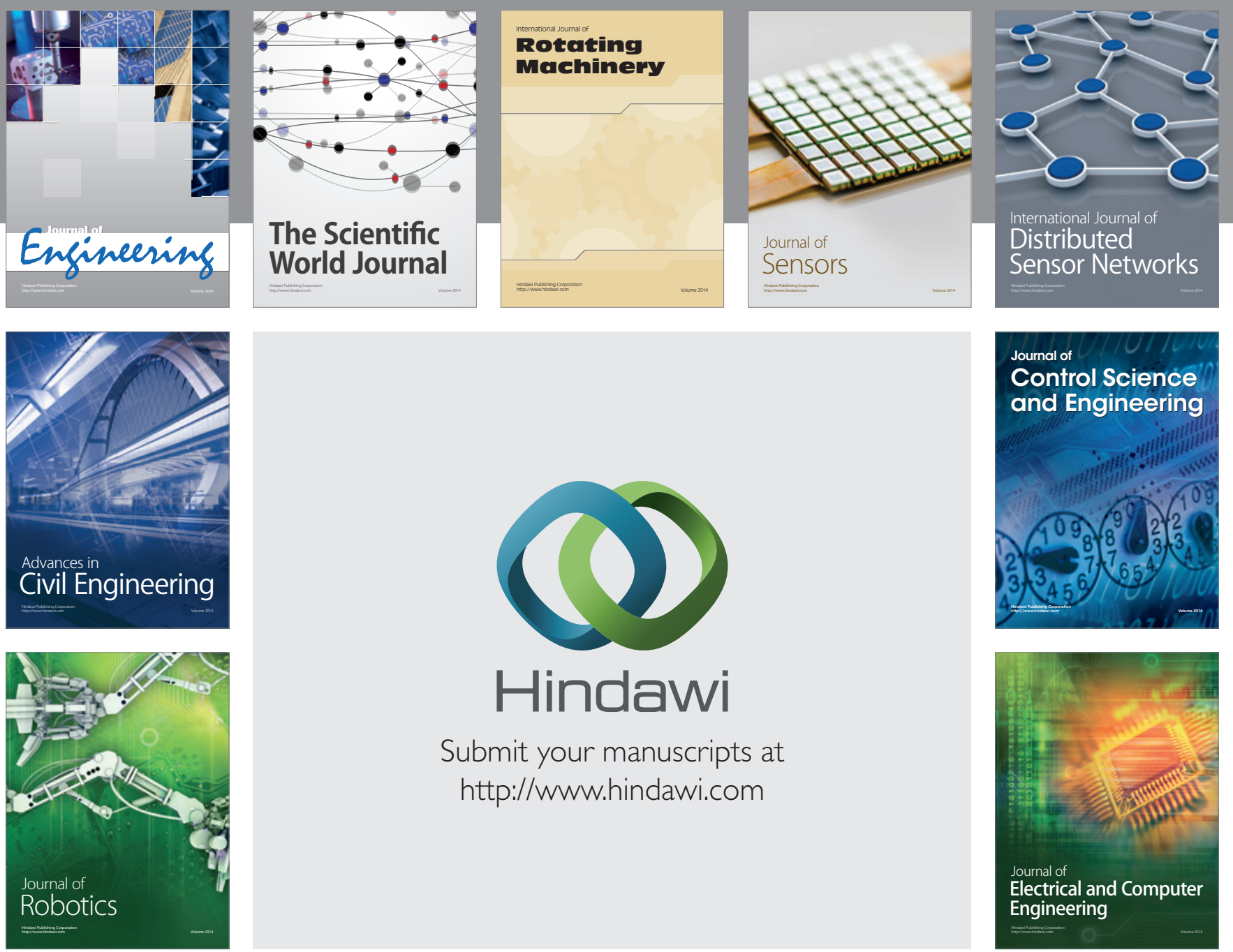

Submit your manuscripts at

http://www.hindawi.com
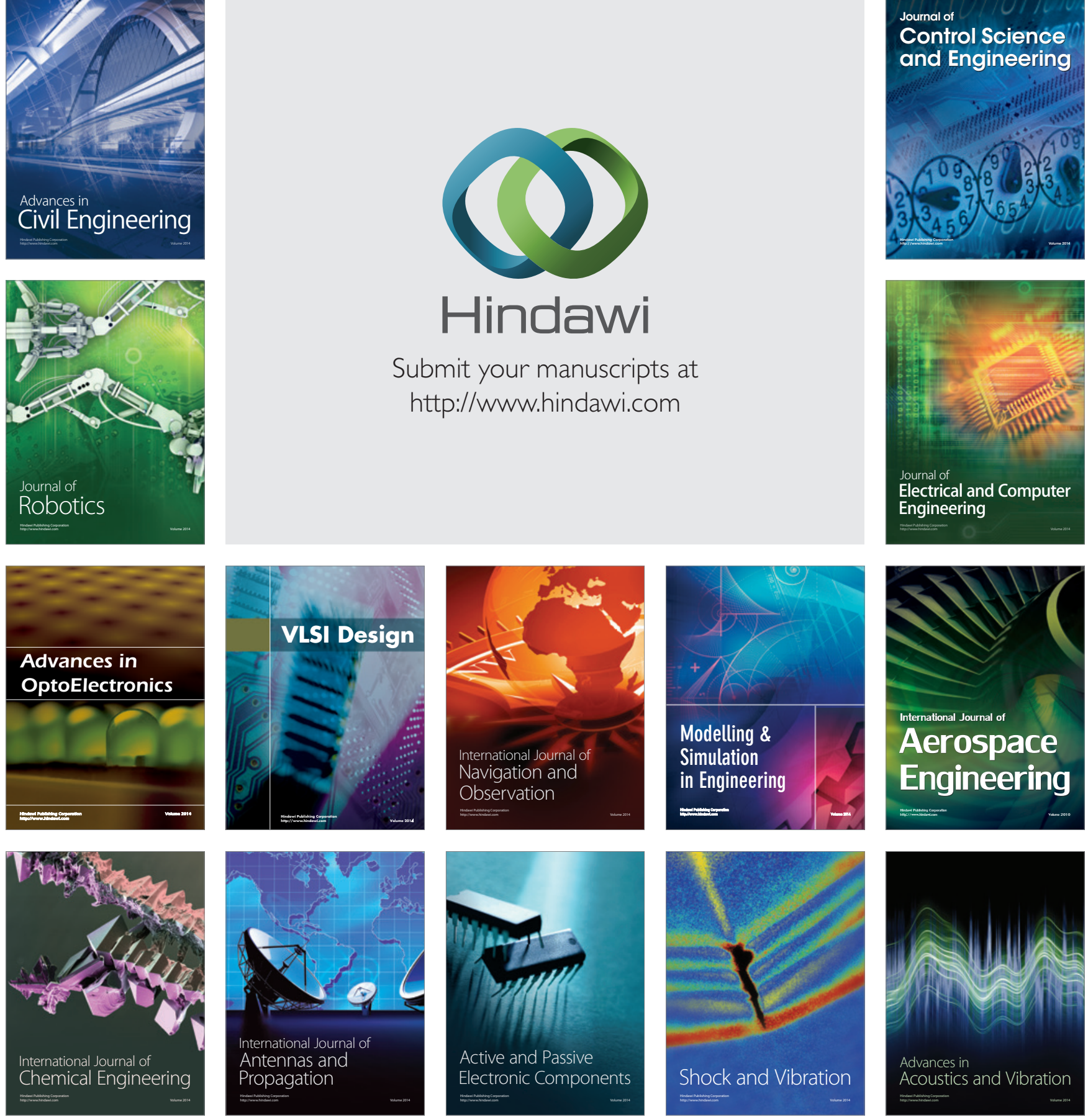